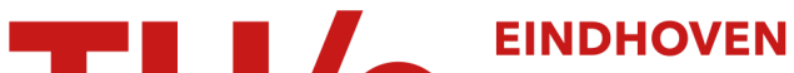 \\ UNIVERSITY OF \\ TECHNOLOGY
}

\section{Discrete prolate spheroidal wave functions and interpolation.}

\section{Citation for published version (APA):}

Delsarte, P., Janssen, A. J. E. M., \& Vries, L. B. (1985). Discrete prolate spheroidal wave functions and interpolation. SIAM Journal on Applied Mathematics, 45(4), 641-650. https://doi.org/10.1137/0145037

DOI:

10.1137/0145037

Document status and date:

Published: 01/01/1985

\section{Document Version:}

Publisher's PDF, also known as Version of Record (includes final page, issue and volume numbers)

\section{Please check the document version of this publication:}

- A submitted manuscript is the version of the article upon submission and before peer-review. There can be important differences between the submitted version and the official published version of record. People interested in the research are advised to contact the author for the final version of the publication, or visit the $\mathrm{DOI}$ to the publisher's website.

- The final author version and the galley proof are versions of the publication after peer review.

- The final published version features the final layout of the paper including the volume, issue and page numbers.

Link to publication

\section{General rights}

Copyright and moral rights for the publications made accessible in the public portal are retained by the authors and/or other copyright owners and it is a condition of accessing publications that users recognise and abide by the legal requirements associated with these rights.

- Users may download and print one copy of any publication from the public portal for the purpose of private study or research.

- You may not further distribute the material or use it for any profit-making activity or commercial gain

- You may freely distribute the URL identifying the publication in the public portal.

If the publication is distributed under the terms of Article 25fa of the Dutch Copyright Act, indicated by the "Taverne" license above, please follow below link for the End User Agreement:

www.tue.nl/taverne

Take down policy

If you believe that this document breaches copyright please contact us at:

openaccess@tue.nl

providing details and we will investigate your claim. 


\title{
DISCRETE PROLATE SPHEROIDAL WAVE FUNCTIONS AND INTERPOLATION*
}

\author{
PH. DELSARTE $\dagger$, A. J. E. M. JANSSEN $\ddagger$ AND L. B. VRIES $\ddagger$
}

\begin{abstract}
We describe an algorithm for the interpolation of burst errors in discrete-time signals that can be modelled as being band-limited. The algorithm correctly restores a mutilated signal that is indeed band-limited. The behavior of the algorithm when applied to signals containing noise or out-of-band components can be analysed satisfactorily with the aid of asymptotic properties of the discrete prolate spheroidal sequences and wave functions. The effect of windowing can also be described conveniently in terms of these sequences and functions.
\end{abstract}

1. Introduction. In this paper we consider discrete-time, real or complex-valued

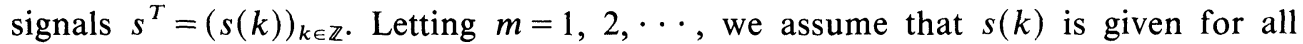
integers $k$ except $k=0,1, \cdots, m-1$. The vector $z^{T}=[s(0), \cdots, s(m-1)]$ of unknown samples is estimated according to the following principle. Assume that $\sum_{k \neq 0,1, \cdots, m-1}|s(k)|^{2}<\infty$. Define the Fourier transform $S$ of $s$ by

$$
S(\theta):=\sum_{k=-\infty}^{\infty} s(k) e^{2 \pi i k \theta} \quad\left(|\theta| \leqq \frac{1}{2}\right)
$$

and let $0<\alpha<1$. Then we choose $z$ so as to minimize

$$
\int_{\alpha / 2 \leqq|\theta| \leqq 1 / 2}|S(\theta)|^{2} d \theta .
$$

This is a finite-dimensional least squares problem in $z$, and the $z$ minimizing (1.2) is given by

$$
\hat{z}=\left(I-M_{0}\right)^{-1}\left(M_{1} t_{1}+M_{2} t_{2}\right),
$$

where $t_{1}^{T}=(s(k))_{k<0}, t_{2}^{T}=(s(k))_{k>m-1}$ and $M_{0}, M_{1}, M_{2}$ are submatrices of the low-pass matrix $M$ :

$$
M=\left[M_{1}\left|M_{0}\right| M_{2}\right]=\left(\frac{\sin \pi(k-l) \alpha}{\pi(k-l)}\right)_{k=0,1, \cdots, m-1,-\infty<l<\infty}
$$

of which the columns with index $l=0,1, \cdots, m-1$ constitute $M_{0}$.

We observe that we can write down the formula (1.3) for $\hat{z}$ for any $s$ for which $M_{1} t_{1}$ and $M_{2} t_{2}$ are well defined; for this to be the case it is not necessary that

$$
\int_{-1 / 2}^{1 / 2}|S(\theta)|^{2} d \theta<\infty .
$$

If e.g. $\theta_{0} \neq \alpha / 2, s(k)=\exp \left(2 \pi i k \theta_{0}\right)$ for $k \in \mathbb{Z}$, then $M_{1} t_{1}$ and $M_{2} t_{2}$ are well defined as conditionally convergent series.

The motivation for choosing $\hat{z}$ as in (1.3) is as follows. The signal $s$ is called band-limited to $\alpha / 2$ if its Fourier transform $S(\theta)$ vanishes for $\alpha / 2 \leqq|\theta| \leqq \frac{1}{2}$. For such an $s$ the $\hat{z}$ of $(1.3)$ equals $z=[s(0), \cdots, s(m-1)]^{T}$ since we have

$$
z=M s=M_{1} t_{1}+M_{0} z+M_{2} t_{2},
$$

\footnotetext{
* Received by the editors May 17, 1983, and in final revised form November 16, 1984.

$\dagger$ Philips Research Laboratory, Brussels, Belgium.

$\ddagger$ Philips Research Laboratories, P.O. Box 80.000, 5600 JA Eindhoven, the Netherlands.
} 
so that indeed $z=\left(I-M_{0}\right)^{-1}\left(M_{1} t_{1}+M_{2} t_{2}\right)$. The purpose of this paper is to find out how stable formula (1.3) is under imprecision of data, such as additive white noise, or the presence of out-of-band components in the signal. We are furthermore interested in the magnitude and nature of the interpolation error $z-\hat{z}$ for such signals. The interpolation method is sufficiently stable only for rather modest values of $m \alpha$, such as $m \alpha \leqq 5$. This can be seen from (2.6) which shows that there are band-limited signals of unit energy whose energy outside the set $\{0,1, \cdots, m-1\}$ is of the order $\exp (-\pi m \alpha)$.

Slepian has derived in [6] asymptotic properties, as $m \rightarrow \infty$, of the eigenvalues and eigenvectors of $M_{0}$ in connection with discrete prolate spheroidal wave functions and sequences. These asymptotic properties are known to be quite accurate, already for values of $m$ as small as 4 or 5 , and can therefore be used for analysing the interpolation method for modest values of $m \alpha$. We refer to Figs. 3-7 of [6].

Let us summarize the contents of this paper. The largest eigenvalue of $M_{0}$ is usually much closer to 1 than the others. This is apparent from [6], Figs. 5-7 (observe that $\alpha=2 W$ ) from which one can see that $1-\lambda_{0}$ is more than 10 (50) times smaller than $1-\lambda_{1}$ for values of $m \alpha$ as small as 2 (3). Hence, there is a strong tendency for $z-\hat{z}\left(=\left(I-M_{0}\right)^{-1}(z-M s)\right.$ according to $(1.3)$ and $\left.(1.5)\right)$ to be a multiple of the eigenvector of $M_{0}$ corresponding to the largest eigenvalue. We show in $\S 3$ that the components of this eigenvector are all of one sign (also see [7, § III] and [5]). Hence, the interpolation error tends to be of one sign; depending on the particular application this property must be considered as a rather unfavourable feature of the algorithm.

In $\S 4$ we study the interpolation error $z-\hat{z}$ when the signal $s$ contains white noise or out-of-band components. We also present a qualitative analysis showing the approximate location of the column index of the largest columns of the infinite matrix $\left(I-M_{0}\right)^{-1}\left[M_{1} \mid M_{2}\right]$ as well as the slow decay to zero of the columns when the column index tends to $\infty$. The reason to study this matrix is formula (1.3), expressing the estimate $\hat{z}$ in terms of the known samples by means of this matrix. In order that the columns of this matrix tend to zero faster one could consider perturbations $\tilde{M}$ of the matrix $M$ in (1.4). These perturbations should have the effect that the columns of $\left(I-\tilde{M}_{0}\right)^{-1}\left[\tilde{M}_{1} \mid \tilde{M}_{2}\right]$ tend to zero faster, while for signals band-limited to $\beta / 2$ (with $\beta$ slightly smaller than $\alpha$ ) still good interpolation results are obtained. The stability of the perturbed method is still largely determined by the distance to 1 of the largest eigenvalues of $\tilde{M}_{0}$. Hence we give in $\S 5$ approximating formulas and plots for the perturbed eigenvalues.

The interpolation method discussed here has been tested on digital audio signals, and the performance of the method for these signals is reported on in [2].

2. Discrete prolate spheroidal sequences and wave functions. We present some facts from [6] in a slightly different notation. We let $M_{0}$ denote the Toeplitz matrix

$$
\left(\frac{\sin \pi(k-l) \alpha}{\pi(k-l)}\right)_{k, l=0,1, \cdots, m-1},
$$

and we order its eigenvalues $\lambda_{k} \equiv \lambda_{k}(m, \alpha)$ decreasingly so that $1>\lambda_{0}>\lambda_{1}>\cdots>$ $\lambda_{m-1}>0$. We denote the corresponding eigenvectors, normalized as in [6], by $v_{k}=$ $\left(v_{k}(r)\right)_{r=0,1, \cdots, m-1}$. As in [6], $v_{k}(r)$ is defined for $r<0, r>m-1$ by

$$
\lambda_{k} v_{k}(r)=\sum_{l=0}^{m-1} \frac{\sin \pi(r-l) \alpha}{\pi(r-l)} v_{k}(l)
$$

and the sequences thus obtained are the discrete prolate spheroidal sequences. 
The discrete prolate spheroidal wave functions $U_{k}(\theta) \equiv U_{k}(m, \alpha ; \theta)$ are given by

$$
U_{k}(\theta)=\varepsilon_{k} \sum_{r=0}^{m-1} v_{k}(r) e^{-\pi i(m-1-2 r) \theta}
$$

where $\varepsilon_{k}$ is 1 or $i$ according as $k$ is even or odd $(k=0,1, \cdots, m-1)$. Among the many formulas satisfied by the $U_{k}$ and $v_{k}$ (see $[6, \S 2.1-2.3$ ), we mention

$$
v_{k}(r)=-\varepsilon_{k}^{-1} \lambda_{k}^{-1} \int_{\alpha / 2 \leqq|\theta| \leqq 1 / 2} U_{k}(\theta) e^{\pi i(m-1-2 r) \theta} d \theta=-\varepsilon_{k}^{-1} \lambda_{k}^{-1} W_{k}\left(\frac{m-1}{2}-r\right)
$$

for $r<0, r>m-1$, where $W_{k}(\tau):=\int_{\alpha / 2 \leqq|\theta| \leqq 1 / 2} U_{k}(\theta) \exp (2 \pi i \tau \theta) d \theta$.

Further properties of the $v_{k}$ and $U_{k}$ can be described in terms of the tridiagonal matrix $D$, defined as

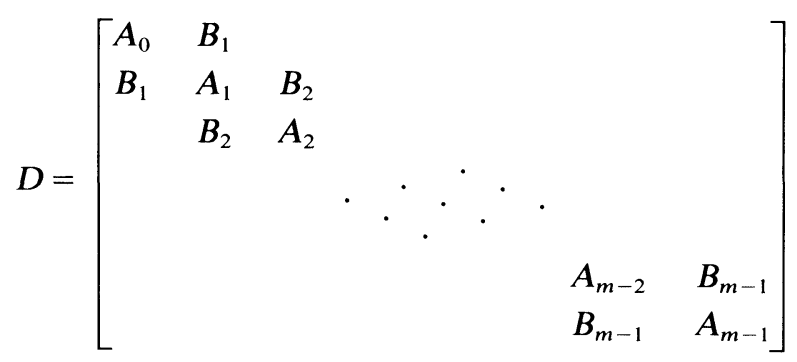

with $A_{k}=((m-1) / 2-k)^{2} \cos \pi \alpha, B_{k}=\frac{1}{2} k(m-k)$. This $D$ has real, distinct eigenvalues $\mu_{k} \equiv \mu_{k}(m, \alpha)$. If we order them as $\mu_{0}>\mu_{1}>\cdots>\mu_{m-1}$, we have

$$
\frac{1}{4 \pi^{2}} \frac{d}{d \theta}\left[(\cos 2 \pi \theta-\cos \pi \alpha) U_{k}^{\prime}(\theta)\right]+\left[\frac{1}{4}\left(m^{2}-1\right) \cos 2 \pi \theta-\mu_{k}\right] U_{k}(\theta)=0
$$

for $k=0,1, \cdots, m-1,|\theta| \leqq \frac{1}{2}$. Furthermore, $M_{0}$ and $D$ commute and have therefore the same eigenvectors. Also, $v_{k}$ is the eigenvector of $D$ with eigenvalue $\mu_{k}$.

In addition, we have the following asymptotic results, for $m \rightarrow \infty$ and fixed $k$ :

$$
\begin{gathered}
1-\lambda_{k} \sim \frac{\sqrt{2 \pi}}{k !}\left[\frac{8 m \sin (\pi \alpha / 2)}{\cos ^{2}(\pi \alpha / 2)}\right]^{k+1 / 2}\left[\frac{\cos (\pi \alpha / 2)}{1+\sin (\pi \alpha / 2)}\right]^{2 m}, \\
\mu_{k}=\frac{1}{4} m^{2}-\left(k+\frac{1}{2}\right) m \sin \pi \frac{\alpha}{2}+O(1), \\
U_{k}(\theta) \sim \begin{cases}a_{k} J_{0}\left(m\left[\frac{\cos \pi \alpha-\cos 2 \pi \theta}{1+\cos \pi \alpha}\right]^{1 / 2}\right), & \left(\frac{\alpha}{2} \leqq \theta \leqq \theta_{m}\right), \\
b_{k} R(\theta) \cos \phi_{k}(\theta), & \left(\theta_{m} \leqq \theta \leqq \frac{1}{2}\right) .\end{cases}
\end{gathered}
$$

Here $J_{0}$ is the Bessel function of 0th order, $\theta_{m}=1 / 2 \pi \arccos \left(\cos \pi \alpha-m^{-3 / 2}\right)$

$$
\begin{gathered}
a_{k}=(-1)^{[k / 2]}\left[\frac{\pi m\left(1-\lambda_{k}\right)}{\cos \pi \alpha / 2)}\right]^{1 / 2}, \quad b_{k}=2(-1)^{[k / 2]}\left[\left(1-\lambda_{k}\right) \sin \pi \frac{\alpha}{2}\right]^{1 / 2}, \\
R(\theta)=[(\cos \pi \alpha-\cos 2 \pi \theta)(1-\cos 2 \pi \theta)]^{-1 / 4}, \\
\phi_{k}(\theta)=\frac{\pi}{4}+\left.\left(F(\sigma)+G_{k}(\sigma)\right)\right|_{\sigma=\alpha / 2} ^{\sigma=\theta},
\end{gathered}
$$




$$
\begin{aligned}
& F(\sigma)=\frac{m}{2} \arcsin \psi_{1}(\sigma), \quad G_{k}(\sigma)=\frac{k+1 / 2}{2} \arcsin \psi_{2}(\sigma), \\
& \psi_{1}(\sigma)=-1+2\left(\frac{\cos \pi \sigma}{\cos (\pi \alpha / 2)}\right)^{2} \\
& \psi_{2}(\sigma)=\left(\frac{1}{\cos (\pi \alpha / 2)}\right)^{2}\left[1+\left(1-\frac{2}{\sin ^{2} \pi \sigma}\right) \sin ^{2} \pi \frac{\alpha}{2}\right]
\end{aligned}
$$

These asymptotic formulas are known to be accurate, also for moderate values of $m$ (see the figures in [6], and Figs. 1 and 2 of the present paper).



FIG. 1. $1-\lambda_{0}(m, \alpha ; \gamma)$ as a function of $\gamma$ for $m=6, \alpha=0.6$ (solid line); the dashed line is the linear approximation obtained from (5.11).

3. Positivity of the zeroth eigenvector of $\boldsymbol{M}_{\mathbf{0}}$. We shall show in this section that $v_{0}$ has positive elements only. We present in addition some observations that concern the conjecture that $\left(I-M_{0}\right)^{-1}$ has positive elements only; one of the authors learned this conjecture from F. A. Grünbaum in 1981.

$v_{0}$ has positive elements. In $\S 2$ it was noted that $v_{k}$ is the eigenvector of $D$ corresponding to the $k$ th eigenvalue. Consider the three-term recurrence relation

$$
B_{k+1} p_{k+1}(x)=\left(x-A_{k}\right) p_{k}(x)-B_{k} p_{k-1}(x)
$$

for $k=0,1, \cdots, m-2$, with the initialization $p_{-1}(x)=0, p_{0}(x)=1$. This generates the polynomials $p_{0}(x), p_{1}(x), \cdots, p_{m-1}(x)$ with $p_{k}(x)$ of degree $k$ and $\lim _{x \rightarrow \infty} p_{k}(x)>0$. In addition, set

$$
p_{m}(x)=\left(x-A_{m-1}\right) p_{m-1}(x)-B_{m-1} p_{m-2}(x) .
$$

It is easy to show from (3.1) and (3.2) that the eigenvalues $\mu_{k}$ of $D$ are the zeros of the polynomial $p_{m}(x)$, and that the corresponding eigenvectors $v_{k}$ of $D$ equal $v_{k}=$ $\sigma_{k}\left[p_{0}\left(\mu_{k}\right), p_{1}\left(\mu_{k}\right), \cdots, p_{m-1}\left(\mu_{k}\right)\right]^{T}$ for some $\sigma_{k} \in \mathbb{R}, \sigma_{k} \neq 0$. In particular, the zeros of $p_{m}(x)$ are real and distinct, and this also holds for the $p_{l}(x)$ with $l<m$. Moreover, the zeros of $p_{l}(x)$ strictly separate those of $p_{l+1}(x)$. This is easily proved from (3.1) and (3.2). Hence, all zeros of all $p_{l}(x)$ with $1 \leqq l \leqq m-1$ lie between $\mu_{m-1}$ and $\mu_{0}$, the extremal zeros of $p_{m}(x)$. Since $\lim _{x \rightarrow \infty} p_{l}(x)>0$ for $1 \leqq l \leqq m-1$ we have $p_{l}\left(\mu_{0}\right)>0$ for $1 \leqq l \leqq m-1$. In view of $v_{0}(l)=\sigma_{0} p_{l}\left(\mu_{0}\right)$ and the normalization of $v_{0}$ we see that all components of $v_{0}$ are positive. (An argument of the same nature yields: 

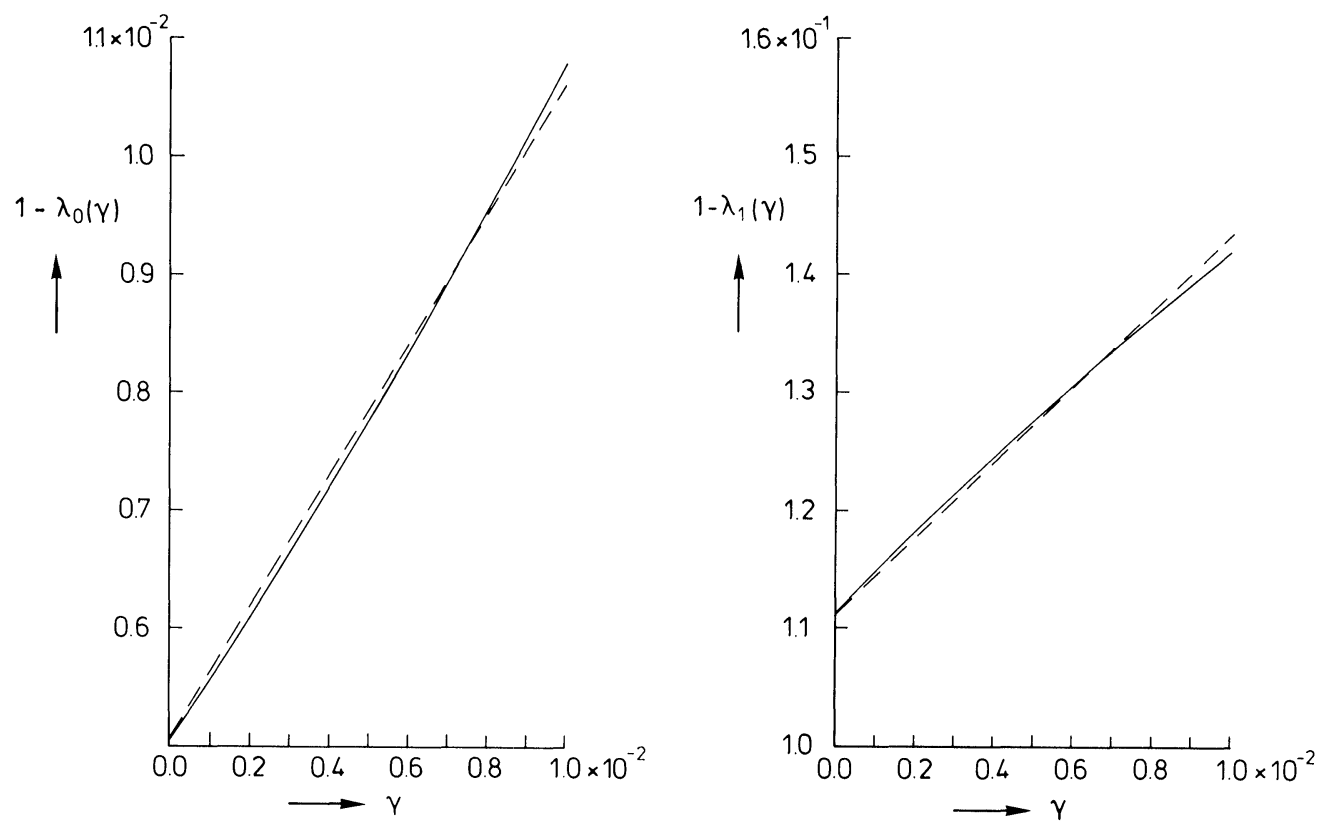

FIG. 2. $1-\lambda_{k}(m, \alpha ; \gamma)$ as a function of $\gamma$ for $m=8, \alpha=0.3, k=0,1$ (solid lines); the dashed lines are the linear approximations obtained from (5.11).

$v_{k}(0), \cdots, v_{k}(m-1)$ has exactly $k$ changes of sign and at most $k$ zeros.) We were kindly informed by one of the referees that a proof of these results can also be derived from $[1, \S 7.11]$.

Conjecture: $\left(I-M_{0}\right)^{-1}$ has positive elements. We have extensive numerical evidence that the matrix $\left(I-M_{0}\right)^{-1}$ has positive elements only. Once this conjecture has been proved, positivity of all components of $v_{0}$ follows from the Perron-Frobenius theorem.

Since $I-M_{0}$ is a symmetric Toeplitz matrix, it makes sense to find a formulation of the positivity condition for $\left(I-M_{0}\right)^{-1}$ in terms of the quantities that appear in the Levinson-Durbin algorithm for the fast solution of a Toeplitz system of linear equations. This can be done as follows. Denote $r_{k}=\sin \pi k \alpha / \pi k$, and let $R^{(p)}=$ $\left(\delta_{k l}-r_{k-1}\right)_{k, l=0,1, \cdots, p-1}, r^{(p)}=\left[-r_{1}, \cdots,-r_{p}\right]^{T}$ for $p=1, \cdots, m$. It follows from elementary matrix theory and $[4, \S C]$, that $\left(I-M_{0}\right)^{-1}$ has positive elements only, if and only if $a(p, p)<0$ for $p=1, \cdots, m$. Here $a(p, p)$ is the $p$ th component of the vector $\left[R^{(p)}\right]^{-1} r^{(p)}$. While it is known that $|a(p, p)|<1$ for all $p$ (cf. [4]), we have numerical evidence that $a(p, p) \rightarrow-\sin (\pi \alpha / 2)$ as $p \rightarrow \infty$. (This formula is accurate for small values of $p$ already.)

In terms of the interpolation algorithm the conjecture admits the following formulation: if $s(k)=0, k \neq-1,0, \cdots, m-1, s(k)$ is unknown for $k=0,1, \cdots, m-1$, and $s(-1)=1$, then $\hat{z}_{m-1}=-a(m, m)>0$.

4. Application to the interpolation algorithm. We analyse the interpolation algorithm of $\S 1$, employing the asymptotic properties of the eigenstructure of $\boldsymbol{M}_{0}$ (accurate as they are for small values of $m \alpha$ already). In the following subsections we consider the effect of additive white noise, and of the presence of out-of-band components, and we study the asymptotic behavior of the norm of the columns of the interpolation matrix $\left(I-M_{0}\right)^{-1}\left[M_{1} \mid M_{2}\right]$. 
4.1. Band-limited signals corrupted by white noise. We first show how additive white noise affects the interpolation. It turns out that the interpolation error must be expected to be pulse-shaped.

THEOREM 4.1. Assume that $s(k)=x(k)+n(k)$, where $x=(x(k))_{k \in \mathbb{Z}}$ is band-limited to $\alpha / 2$ and $(n(k))_{k \in \mathbb{Z}}$ is additive white noise with variance $\sigma^{2}$. There are random variables $p_{k}, k=0,1, \cdots, m-1$ with $\mathscr{E}\left[p_{k}\right]=0, \mathscr{E}\left[p_{k} p_{l}\right]=\sigma^{2} \lambda_{k}\left(1-\lambda_{k}\right)^{-1} \delta_{k l}$, such that $z_{0}-\hat{z}=$ $\sum_{k=0}^{m-1} p_{k} v_{k}$, where $z_{0}^{T}=[x(0), x(1), \cdots, x(m-1)]$.

Proof. We may assume that $z_{0}=0$. We let $t^{T}=\left[t_{1}^{T}|0| t_{2}^{T}\right]$ so that

$$
M_{1} t_{1}+M_{2} t_{2}=M t, \quad \hat{z}=\left(I-M_{0}\right)^{-1} M t=\sum_{k=0}^{m-1}\left(1-\lambda_{k}\right)^{-1}\left(M t, v_{k}\right) v_{k}
$$

When $q_{k}:=\left(M t, v_{k}\right)$ it can be shown from the definition of the $v_{k}$ and the orthogonality relations satisfied by the $v_{k}$ (over the ranges $\{0,1, \cdots, m-1\}$ and $\{\cdots,-2,-1\} \cup$ $\{m, m+1, \cdots\}$, see [6]) that $\mathscr{E}\left[q_{k}\right]=0, \mathscr{E}\left[q_{k} q_{l}\right]=\sigma^{2} \lambda_{k}\left(1-\lambda_{k}\right) \delta_{k l}$. Hence, the theorem is proved with $p_{k}=-\left(1-\lambda_{k}\right)^{-1} q_{k}$.

Note. In view of the asymptotic properties of the $\lambda_{k}$ as given in (2.6) it follows that usually $1-\lambda_{0}$ is much smaller than $1-\lambda_{1}, 1-\lambda_{1}$ is much smaller than $1-\lambda_{2}$, and so on. Hence, in the sum $\sum_{k=0}^{m-1} p_{k} v_{k}$ the term with $k=0$ is usually dominant. It is now clear from the result of $\S 3$ that adding white noise to band-limited signals often results in one-sided interpolation errors. Compare Fig. 2 in [2].

4.2. Signals containing out-of-band components. The following theorem can be used to determine the effect of out-of-band components on the interpolation result. As in the previous subsection, the interpolation errors must be expected to be pulseshaped.

THEOREM 4.2. Let $|\theta| \leqq \frac{1}{2},|\theta| \neq \alpha / 2$, and let $s_{\theta}^{T}=(\exp (2 \pi i k \theta))_{k \in \mathbb{Z}}$ be partitioned in the usual way as $\left[t_{1 \theta}^{T}\left|z_{\theta}^{T}\right| t_{2 \theta}^{T}\right]$. Then we have $z_{\theta}-\hat{z}_{\theta}=0$ or $\sum_{k=0}^{m-1} c_{k}(\theta) v_{k}$ according as $|\theta|<\alpha / 2$ or $>\alpha / 2$, where $c_{k}(\theta)=\left(1-\lambda_{k}\right)^{-1} \varepsilon_{k}^{-1} \times \exp (\pi i(m-1) \theta) U_{k}(\theta)$.

Proof. We have (see $\S 1)\left(M s_{\theta}\right)(r)=s_{\theta}(r)=z_{\theta}(r)$ or 0 according as $|\theta|<\alpha / 2$ or $>\alpha / 2$. Hence $\hat{z}_{\theta}=z_{\theta}$ or $-\left(I-M_{0}\right)^{-1} M_{0} z_{\theta}$ according as $|\theta|<\alpha / 2$ or $>\alpha / 2$. For $|\theta|>\alpha / 2$ we have $z_{\theta}-\hat{z}_{\theta}=\sum_{k=0}^{m-1}\left(1-\lambda_{k}\right)^{-1}\left(z_{\theta}, v_{k}\right) v_{k}$, and by $(2.2)$ we have $\left(z_{\theta}, v_{k}\right)=$ $\varepsilon_{k}^{-1} \exp (\pi i(m-1) \theta) U_{k}(\theta)$. This proves the theorem.

Note. It follows from (2.8) that $U_{k}$ asymptotically (for small $k$ ) is rapidly oscillating in the set $\alpha / 2 \leqq|\theta| \leqq \frac{1}{2}$, with a slowly varying amplitude $\left|b_{k} R(\theta)\right|$ on a large part of that set. Since $b_{k}$ is proportional to $\left(1-\lambda_{k}\right)^{1 / 2}$, we see that the most important term in the $\operatorname{sum} \sum_{k=0}^{m-1} c_{k}(\theta) v_{k}$ is usually the one with $k=0$. This implies that the interpolation error $z_{\theta}-\hat{z}_{\theta}$ tends to be one-sided. Compare Fig. 3 in [2].

4.3. Asymptotic behaviour of the columns of the interpolation matrix. We conclude this section by indicating roughly the behaviour of the columns $\left(I-M_{0}\right)^{-1} y^{(r)}$ of the matrix $\left(I-M_{0}\right)^{-1}\left[M_{1} \mid M_{2}\right]$, where

$$
y^{(r)}=\left(\frac{\sin \pi(r-l) \alpha}{\pi(r-l)}\right)_{l=0,1, \cdots, m-1}^{T} .
$$

By the definition of the $v_{k}$ 's we have

$$
\left(I-M_{0}\right)^{-1} y^{(r)}=\sum_{k=0}^{m-1}\left(1-\lambda_{k}\right)^{-1} \lambda_{k} v_{k}(r) v_{k} .
$$

Note that the contribution over the set $\{r \mid r<0, r>m-1\}$ of the square of the norm 
of the $k$ th term in this sum equals

$$
\left(\sum_{r<0}+\sum_{r>m-1}\right)\left(1-\lambda_{k}\right)^{-2} \lambda_{k}^{2}\left|v_{k}(r)\right|^{2}=\left(1-\lambda_{k}\right)^{-1} \lambda_{k}
$$

(see [6]), and this is clearly largest for small $k$. Hence, we must find information about the asymptotic behavior of $v_{k}(r)$ for $k$ small and $r<0$ or $r>m-1$. To that end we use the expression (2.3) of $v_{k}(r)$ in terms of $W_{k}$.

The asymptotic behavior of $W_{k}(\tau)$ as $m$ gets large can be determined from the asymptotic behavior of $U_{k}(\theta)$ in the range $\alpha / 2 \leqq \theta \leqq \frac{1}{2}$ which is given by (2.8) and (2.6). Ignoring the contribution of the set $\alpha / 2 \leqq \theta \leqq \theta_{m}$, we obtain an approximation of $W_{k}(\tau)$ by considering $Z_{k}(\tau)+(-1)^{k} Z_{k}(\tau)$, where

$$
Z_{k}(\tau)=b_{k} \int_{\alpha / 2 \leqq \theta \leqq 1 / 2} e^{2 \pi i \tau \theta} R(\theta) \cos \phi_{k}(\theta) d \theta
$$

with $b_{k}, R$ and $\phi_{k}$ given by (2.9)-(2.13).

The integral in (4.3) is fit for application of the stationary phase method. We can write the formula $(2.11)$ for $\phi_{k}(\theta)$ as

$$
\phi_{k}(\theta)=\frac{\pi}{4}+\int_{\alpha / 2}^{\theta}\left(F^{\prime}(\sigma)+G_{k}^{\prime}(\sigma)\right) d \sigma
$$

By employing some trigonometric identities it is seen that

$$
F^{\prime}(\sigma)=\frac{-m \pi \sin \pi \sigma}{\sqrt{\sin ^{2} \pi \sigma-\sin ^{2}(\pi \alpha / 2)}}, \quad G_{k}^{\prime}(\sigma)=\frac{\pi(k+1 / 2) \sin (\pi \alpha / 2) / \sin \pi \sigma}{\sqrt{\sin ^{2} \pi \sigma-\sin ^{2}(\pi \alpha / 2)}} .
$$

Let $\tau>0$. According to the stationary phase principle the largest contribution to the integral (4.3) comes from the $\theta$-region where $\phi_{k}^{\prime}(\theta)$ is close to $\pm 2 \pi \tau$, i.e. where $F^{\prime}(\theta)+G_{k}^{\prime}(\theta)$ is close to $\pm 2 \pi \tau$. Now when $k$ is small compared to $m$, we may ignore $G_{k}$. It turns out that $F^{\prime}$ increases on $\left[\alpha / 2, \frac{1}{2}\right]$ from $-\infty$ to $-m \pi /(\cos (\pi \alpha / 2))$, and $F^{\prime \prime}\left(\frac{1}{2}\right)=0$. Hence, it can be expected that $\left|Z_{k}(\tau)\right|$ is close to its maximum value when $\tau$ is such that $F^{\prime}\left(\frac{1}{2}\right)=-2 \pi \tau$, i.e. $\tau=m /(2 \cos (\pi \alpha / 2))$. Furthermore there are no solutions $\theta$ of the equation $F^{\prime}(\theta)=-2 \pi \tau$ when $0<\tau<m /(2 \cos (\pi \alpha / 2))$, hence it must be expected that $\left|Z_{k}(\tau)\right|$ is small for $\tau$ in the vicinity of 0 . Finally, when $|\tau|>$ $m /(2 \cos (\pi \alpha / 2))$, the contribution to (4.3) of the $\theta$-region where $F^{\prime}(\theta)$ is close to $-2 \pi \tau$ is of the order $R\left(\theta_{0}\right)\left|F^{\prime \prime}\left(\theta_{0}\right)\right|^{-1 / 2}$, where $\theta_{0}$ is such that $F^{\prime}\left(\theta_{0}\right)=-2 \pi \tau$. Using the explicit formulas for $R$ and $F$ and noting that $\theta_{0}-\alpha / 2=O\left(m \tau^{-1}\right)$, we find that $\left|Z_{k}(\tau)\right|=O\left(b_{k} m^{1 / 2} \tau^{-1}\right)$ when $\tau$ gets large and $k$ is small compared to $m$.

This provides sufficient information for getting an idea how $v_{k}(r)$ behaves for small $k$ and $r<0$ or $r>m-1$. As a result we see from (4.1) that $\left\|\left(I-M_{0}\right)^{-1} y^{(r)}\right\|$ can be expected to be largest for $|r-(m-1) / 2| \sim m /(2 \cos (\pi \alpha / 2))$. It was found by computer simulations that this estimate is quite accurate, also for moderate values of $m$. Furthermore, it is seen that the decay rate of $v_{k}(r)$ is not impressive: it can be expected that $v_{k}(r)=O\left(r^{-1}\right)$ as $r \rightarrow \infty$.

5. Perturbing the low-pass matrix $M$. In this section we replace the matrix $M$ of (1.4) by a windowed version of it so as to obtain an interpolation matrix whose columns tend to zero faster (see 3 rd subsection of $\S 4$ ). This windowing is achieved by replacing the numbers $r_{k}=\sin \pi k \alpha / \pi k$ in (1.4) by $r_{k}(\gamma)=r_{k} W\left(\gamma^{1 / 2} k\right)$, where $W$ is a smooth even function on $\mathbb{R}$ with $W(x) \rightarrow 0$ when $x \rightarrow \infty$, and $\gamma>0$ is small. One should choose $W$ and $\gamma$ such that the interpolation results are satisfactory for all signals band-limited 
to $\beta / 2$, where $\beta$ is only slightly smaller than $\alpha$. To judge the stability of the interpolation method thus obtained, one should know how the eigenvalues of the windowed version of the matrix $M_{0}$ behave as a function of $\gamma$. It turns out that for Gaussian $W$ one can derive, for the largest eigenvalues, perturbation series the first 2 terms of which provide an accurate approximation in the relevant $\gamma$-range.

We start with the following observation.

TheOrem 5.1. Assume $\sum_{k=-\infty}^{\infty}|s(k)|^{2}<\infty$. Let $Q=(q(k-l))_{k=0,1, \cdots, m-1,-\infty<l<\infty}$ where $q(-k)=q(k)$ for $k \in \mathbb{Z}, \sum_{k=-\infty}^{\infty}|q(k)|^{2}<\infty$ and $Q_{0}=(q(k-l))_{k, l=0,1, \cdots, m-1}$ is positive definite. Partition $Q=\left[Q_{1}\left|Q_{0}\right| Q_{2}\right], \quad s^{T}=\left[t_{1}^{T}|z| t_{2}^{T}\right], \quad$ and let $p(\theta)=$ $\sum_{k=-\infty}^{\infty} q(k) \exp (-2 \pi i k \theta)$. Then $\hat{z}:=-Q_{0}^{-1}\left(Q_{1} t_{1}+Q_{2} t_{2}\right)$ minimizes the integral

$$
I(a):=\int_{-1 / 2}^{1 / 2} p(\theta)\left|\sum_{k=-\infty}^{\infty} \hat{s}(k) e^{2 \pi i k \theta}\right|^{2} d \theta
$$

as a function of $a=[a(0), a(1), \cdots, a(m-1)]^{T}$, where $\hat{s}(k)=a(k)$ or $s(k)$ according as $0 \leqq k \leqq m-1$ or not.

Proof. Insert in the integral for $I(a)$ the definition of $p$ and write out the integrand as a triple sum. Performing the integration and using $\int_{-1 / 2}^{1 / 2} \exp (2 \pi i k \theta) d \theta=\delta_{k 0}$ for integer $k$, one gets

$$
I(a)=\left(Q_{0} a, a\right)+2 \operatorname{Re}\left[\left(a, Q_{1} t_{1}+Q_{2} t_{2}\right)\right]+C,
$$

where $C$ is a constant determined by $t_{1}$ and $t_{2}$. The proof is easily completed now.

Now consider the windowed matrix

$$
M(\gamma)=\left[M_{1}(\gamma)\left|M_{0}(\gamma)\right| M_{2}(\gamma)\right]=\left(r_{k-l}(\gamma)\right)_{k=0,1, \cdots, m-1,-\infty<l<\infty} .
$$

The function $p(\theta) \equiv p(\theta ; \gamma)$ of Theorem 5.1 is given by

$$
p=\left(1-\chi_{\alpha}\right) * \psi_{\gamma}
$$

where $\chi_{\alpha}(\theta)=0(|\theta| \leqq \alpha / 2), \chi_{\alpha}(\theta)=\mid\left(\alpha / 2 \leqq|\theta| \leqq \frac{1}{2}\right)$, the asterisk denotes convolution for periodic functions of period 1 , and

$$
\psi_{\gamma}(\theta)=\sum_{k=-\infty}^{\infty} W\left(\gamma^{1 / 2} k\right) e^{2 \pi i k \theta}
$$

According to Szegö's limit theorem, the eigenvalue distribution of the matrix $Q_{0}$ in Theorem 5.1 as $m \rightarrow \infty$ is asymptotically equal to the value distribution of the function $p$. Hence, the largest eigenvalues of $M_{0}(\gamma)$ in (5.3) tend to decrease with increasing $\gamma>0$ (provided that $\psi_{\gamma} \geqq 0$ ). Moreover $\left|r_{k}(\gamma)\right|$ is a decreasing function of $\gamma>0$. Hence, there is definitely a tendency for the columns of $\left(I-M_{0}(\gamma)\right)^{-1}\left[M_{1}(\gamma) \mid M_{2}(\gamma)\right]$ to decrease in magnitude when $\gamma$ increases and also to tend to zero faster when the column index tends to $\infty$. The price to be paid is that when $\gamma$ is too large the function $p$ is too large around $\theta=0$ which results in unsatisfactory restoration of signals with significant low-frequency components. More specific numbers and figures are presented at the end of this section for the case of a Gaussian window $W$.

We shall now use some perturbation theory to find approximations for $\lambda_{k} \equiv$ $\lambda_{k}(m, \alpha ; \gamma)$, the $k$ th eigenvalue of $M_{0}(\gamma)$. To that end we assume that $W$ has a power series expansion around 0 ,

$$
W(x)=\sum_{n=0}^{\infty} \frac{W^{(2 n)}(0)}{(2 n) !} x^{2 n}
$$


It is a rather immediate consequence of the theory in [3, Chap. II, $\S 2$ that, for small $\gamma>0$,

$$
\lambda_{k}(\gamma)=\sum_{n=0}^{\infty} c_{k, n} \gamma^{n}
$$

where the coefficients $c_{k, n}$ can be expressed in terms of the unperturbed $\lambda_{k}$ and the numbers $\left(M_{0}^{(\nu)} v_{j}, v_{i}\right)$, where

$$
M_{0}^{(\nu)}=\frac{W^{(2 \nu)}(0)}{(2 \nu) !}\left((k-l)^{2 \nu} r_{k-l}\right)_{k, l=0,1, \cdots, m-1}
$$

For example, the first three terms of the expansion for $\lambda_{k}(\gamma)$ are

$$
\lambda_{k}+\gamma\left(M_{0}^{(1)} v_{k}, v_{k}\right)+\gamma^{2}\left[\left(M_{0}^{(2)} v_{k}, v_{k}\right)-\sum_{j \neq k} \frac{\left|\left(M_{0}^{(1)} v_{j}, v_{k}\right)\right|^{2}}{\lambda_{j}-\lambda_{k}}\right] .
$$

It is possible to express the coefficients $c_{k, n}$ in terms of the $U_{k}$ 's. Indeed, it is not hard to show that

$$
\left(M_{0}^{(\nu)} v_{j}, v_{i}\right)= \begin{cases}\frac{2 W^{(2 \nu)}(0)}{\left(4 \pi^{2}\right)^{\nu}(2 \nu) !}(-1)^{\nu}\left[\frac{d^{2 \nu-1}}{d \theta^{2 \nu-1}} U_{j} U_{i}\right]\left(\frac{\alpha}{2}\right), & j-i \text { even } \\ 0, & j-i \text { odd } .\end{cases}
$$

In (5.10) the derivatives of the $U_{k}$ 's at $\alpha / 2$ can be expressed in terms of $\mu_{k}$ and $U_{k}(a / 2)$ by evaluating and differentiating (2.5) repeatedly at $\theta=\alpha / 2$ (where $\cos 2 \pi \theta-\cos \pi \alpha=$ 0 ). We thus find the first order approximation ( $k$ small compared to $m$ ) for $\lambda_{k}(\gamma)$ :

$$
\begin{aligned}
\lambda_{k}+ & \frac{m \gamma W^{(2)}(0)}{2 \sin (\pi \alpha / 2) \cos ^{2}(\pi \alpha / 2)} \\
& \cdot\left[\frac{1}{2}\left(m^{2}-1\right) \sin ^{2} \pi \frac{\alpha}{2}-\left(k+\frac{1}{2}\right) m \sin \pi \frac{\alpha}{2}+\frac{1}{4}\right]\left(1-\lambda_{k}\right) .
\end{aligned}
$$

We finally present some results for the window function $W(x)=\exp \left(-x^{2}\right)$. With respect to the choice of $\gamma$ we note a trade-off between faster decrease of the columns of the interpolation matrix and deterioration of the interpolation results for in-band signals with spectral energy close to the bounds of the interval $[-\alpha / 2, \alpha / 2]$. For values of $m \alpha$ in the range $[2,4]$ we noticed that making the elements $m_{k l}(\gamma)$ of the interpolation matrix $\left(I-M_{0}(\gamma)\right)^{-1} M(\gamma)$ vanish up to 3 decimal places for $k=0,1, \cdots, m-1$, $l \leqq-100$ or $l \geqq 200+m-1$ required a $\gamma$ in the range $\left[10^{-3}, 10^{-2}\right]$. Taking $\gamma$ larger than $10^{-2}$ resulted in unsatisfactory interpolation results for exponentials $s(k)=\exp (2 \pi i k \theta)$ with $\beta / 2 \leqq|\theta| \leqq \alpha / 2$ with $\beta$ up to over $20 \%$ smaller than $\alpha$. Figures 1,2 show graphs of $1-\lambda_{k}(m, \alpha ; \gamma)$ as a function of $\gamma$ in the range $\left[0,10^{-2}\right]$. In this range and for the values of $m$ and $\alpha$ as chosen in the figures, the linear approximation (5.11) turns out to be quite convincing. This further underlines the accuracy of Slepian's asymptotic results.

\section{REFERENCES}

[1] J. N. Franklin, Matrix Theory, Prentice-Hall, Englewood Cliffs, NJ, 1968.

[2] A. J. E. M. JANSSEN AND L. B. VRIES, Interpolation of band-limited discrete-time signals by minimizing out-of-band energy, Proc. IEEE Internat. Conf. ASSP, 1984.

[3] T. Kato, Perturbation Theory for Linear Operators, Springer-Verlag, New York, 1966. 
[4] J. MAKhOul, Linear prediction: a tutorial review, Proc. IEEE, 63 (1975), pp. 561-580.

[5] A. Papoulis AND M. S. Bertran, Digital filtering and prolate functions, IEEE Trans. Circuit Theory, CT-19 (1972), pp. 674-681.

[6] D. SLEPIAN, Prolate spheroidal wave functions, Fourier analysis, and uncertainty - V: The discrete case, Bell Syst. Tech. J., 57 (1978), pp. 1371-1429.

[7] D. J. THOMSON, Spectrum estimation and harmonic analysis, Proc. IEEE, 70 (1982), pp. 1055-1096. 